\title{
New Insights Into Lightning Return-Stroke Models With Specified Longitudinal Current Distribution
}

\author{
Grzegorz Maslowski, Member, IEEE, and Vladimir A. Rakov, Fellow, IEEE
}

(Invited Paper)

\begin{abstract}
We show that any lightning return-stroke model with specified longitudinal current distribution can be expressed, using the appropriate continuity equation, in terms of two current components, propagation current and corona current. For lumped-current-source (LCS)-type (transmission-line-type) models, the actual corona current is unipolar and directed radially out of the channel core, while for distributed-current-source (DCS)type (traveling-current-source-type) models, it is also unipolar but directed into the channel core. The conversion between LCS and DCS return-stroke models alters the actual corona current (if any) of the model. A new formulation for LCS lightning return-stroke models is proposed using corona current and propagation current concepts. This formulation expresses the longitudinal current at height $z^{\prime}$ as the channel-base current minus the overall longitudinal current change from ground surface to $z^{\prime}$. Dynamics of lightning-channel corona sheath surrounding the thin channel core are examined based on LCS and DCS models.
\end{abstract}

Index Terms-Continuity equation, corona sheath, distributed sources, lightning, lumped source, return-stroke model.

\section{INTRODUCTION}

A LIGHTNING return-stroke model with specified longitudinal current distribution (an engineering return-stroke model) is usually defined as an equation relating the longitudinal channel current $i\left(z^{\prime}, t\right)$ at any height $z^{\prime}$ and any time $t$ to the current $i(0, t)$ at the channel origin, $z^{\prime}=0$ (e.g., [1]), i.e.,

$$
i\left(z^{\prime}, t\right)=u\left(t-z^{\prime} / v_{f}\right) P\left(z^{\prime}\right) i\left(0, t-z^{\prime} / v\right)
$$

where $u$ is the Heaviside function equal to unity for $t \geq$ $z^{\prime} / v_{f}$ and zero otherwise, $P\left(z^{\prime}\right)$ is the height-dependent current-attenuation factor introduced in [2], $v_{f}$ is the upwardpropagating front speed, and $v$ is the current-wave propagation speed. An equivalent formulation in terms of the line charge density $\rho\left(z^{\prime}, t\right)$ expressed as the sum of transferred and deposited charge density components was proposed in [3].

We will show in this paper that any lightning return-stroke model with specified longitudinal current distribution can be expressed, using the appropriate continuity equation, in terms of two current components, propagation current and corona current. A new formulation for LCS lightning return-stroke mod-

Manuscript received October 7, 2008. First published May 2, 2009; current version published August 21, 2009.

G. Maslowski was with the Department of Electrical and Computer Engineering, University of Florida, Gainesville, FL 32611 USA. He is now with the Department of Electrical and Computer Engineering, Rzeszow University of Technology, Rzeszow 35-959, Poland (e-mail: maslowski@ prz.edu.pl).

V. A. Rakov is with the Department of Electrical and Computer Engineering, and the International Center for Lightning Research and Testing, University of Florida, Gainesville, FL 32611 USA (e-mail: rakov@ece.ufl.edu).

Digital Object Identifier 10.1109/TEMC.2009.2017200 els is proposed using corona current and propagation current concepts. Dynamics of lightning-channel corona sheath surrounding the thin channel core are examined based on the LCS and distributed-current-source (DCS) models.

The lightning channel corona dynamics directly influence the distribution of longitudinal current along the channel. This current is needed for calculation of lightning electromagnetic fields and lightning-induced effects in various circuits. Nonrealistic implied specifications of corona dynamics in the transmission line model (TL) and modified transmission line model with exponential current decay with height (MTLE) lead to nonrealistic model-predicted electric fields within some tens of meters of the lightning channel (e.g., [3]). Furthermore, lightning electromagnetic environment in the immediate vicinity (e.g., [16]) of the strike point is largely determined by processes in the corona sheath.

\section{CONTINUITY EQUATIONS FOR LCS AND DCS RETURN-STROKE MODELS}

Maslowski and Rakov [4] showed that LCS models, those implying an LCS at the lightning channel base, can be described by the following equation:

$$
i_{\text {cor }}^{\prime}\left(z^{\prime}, t\right)+\frac{1}{v} \frac{\partial i\left(z^{\prime}, t\right)}{\partial t}=-\frac{\partial i\left(z^{\prime}, t\right)}{\partial z^{\prime}}
$$

where $i_{\text {cor }}^{\prime}$ is the radial corona current per unit length injected from the central core into the corona sheath and $v$ is the upwardpropagating current-wave speed. Previously, Cooray [6] derived the similar equation for DCS models

$$
i_{\text {cor }}^{\prime}\left(z^{\prime}, t\right)-\frac{1}{c} \frac{\partial i\left(z^{\prime}, t\right)}{\partial t}=-\frac{\partial i\left(z^{\prime}, t\right)}{\partial z^{\prime}}
$$

where $i_{\text {cor }}^{\prime}$ is the radial corona current per unit length injected into the lightning channel core and $c$ is the downwardpropagating current-wave speed equal to the speed of light. Expressions (2) and (3) can be viewed as manifestations of continuity equations for LCS- and DCS-type models, respectively. This can be demonstrated as follows. The total charge per unit length for LCS-type models can be expressed, for $t \geq z^{\prime} / v$, as

$$
\rho\left(z^{\prime}, t\right)=\frac{i\left(z^{\prime}, t\right)}{v}-\frac{d P\left(z^{\prime}\right)}{d z^{\prime}} \int_{z^{\prime} / v}^{t} i\left(0, \tau-z^{\prime} / v\right) d \tau
$$

where the first term of (4) is equal to the transferred charge density $\rho_{\text {tran }}\left(z^{\prime}, t\right)$ and the second term is equal to the deposited charge density $\rho_{\text {dep }}\left(z^{\prime}, t\right)$ at the specified channel segment. These two components of charge density and (4) have been 
introduced in [3]. The time derivative of (4) is

$$
\frac{\partial \rho\left(z^{\prime}, t\right)}{\partial t}=\frac{1}{v} \frac{\partial i\left(z^{\prime}, t\right)}{\partial t}-\frac{d P\left(z^{\prime}\right)}{d z^{\prime}} i\left(0, t-z^{\prime} / v\right)
$$

where the second term on the right-hand side defines the unipolar corona current per unit channel length $i_{\text {cor }}^{\prime}$ for LCS-type models, which was derived in [7] based on the charge conservation principle

$$
i_{\text {cor }}^{\prime}\left(z^{\prime}, t\right)=-\frac{d P\left(z^{\prime}\right)}{d z^{\prime}} i\left(0, t-z^{\prime} / v\right) .
$$

It means that the corona current $i_{\text {cor }}^{\prime}$ per unit channel length is equal to the time derivative of the deposited charge density $\rho_{\text {dep }}$, and (4) can be written in the following equivalent form:

$$
\rho\left(z^{\prime}, t\right)=\frac{i\left(z^{\prime}, t\right)}{v}+\int_{z^{\prime} / v}^{t} i_{\text {cor }}^{\prime}\left(z^{\prime}, t\right) d \tau .
$$

Substituting (6) into (5) and then (5) into (2), one can obtain the general form of the continuity equation

$$
\frac{\partial \rho\left(z^{\prime}, t\right)}{\partial t}=-\frac{\partial i\left(z^{\prime}, t\right)}{\partial z^{\prime}}
$$

In the same way one can derive (8) considering (3), with the time derivative of the total charge per unit length for DCS-type models being expressed for $t \geq z^{\prime} / v$ as [7]

$$
\rho\left(z^{\prime}, t\right)=-\frac{i\left(z^{\prime}, t\right)}{c}+\int_{z^{\prime} / v}^{t} i_{\text {cor }}^{\prime}\left(z^{\prime}, t\right) d \tau .
$$

Thus, (2) and (3) represent the continuity equations for LCSand DCS-type models, respectively.

\section{NEW FORMULATION FOR LCS MODELS}

The spatial integral of (2) evaluated from 0 to $z^{\prime}\left(z^{\prime}\right.$ is behind the return stroke front) is given by

$$
-\int_{0}^{z^{\prime}} \frac{\partial i(\xi, t)}{\partial \xi} d \xi=\int_{0}^{z^{\prime}} \frac{1}{v} \frac{\partial i(\xi, t)}{\partial t} d \xi+\int_{0}^{z^{\prime}} i_{\mathrm{cor}}^{\prime}(\xi, t) d \xi
$$

from which it follows that

$$
i\left(z^{\prime}, t\right)=i(0, t)-\int_{0}^{z^{\prime}} \frac{1}{v} \frac{\partial i(\xi, t)}{\partial t} d \xi-\int_{0}^{z^{\prime}} i_{\text {cor }}^{\prime}(\xi, t) d \xi
$$

Note that the second term on the right-hand side of (11) is nonzero for LCS-type models only for the case of finite speed $v$ (for $v \rightarrow \infty$, this term vanishes) of the upward-propagating current wave, which is assumed to be the same as the upwardpropagating return-stroke front speed $v_{f}$. The kernel of second term's integral is equal to the time derivative of the transferred charge density $\rho_{\operatorname{tran}}\left(z^{\prime}, t\right)=i\left(z^{\prime}, t\right) / v$ introduced in [3]. We define this kernel as the propagation current per unit channel length, which represents the longitudinal current change (per unit length) due to upward propagation delay, i.e.,

$$
i_{\text {prop }}^{\prime}\left(z^{\prime}, t\right)=\frac{\partial \rho_{\operatorname{tran}}\left(z^{\prime}, t\right)}{\partial t}=\frac{1}{v} \frac{\partial i\left(z^{\prime}, t\right)}{\partial t} .
$$

For $t \geq z^{\prime} / v$, we get

$$
i_{\text {prop }}^{\prime}\left(z^{\prime}, t\right)=\frac{P\left(z^{\prime}\right)}{v} \frac{\partial i\left(0, t-z^{\prime} / v\right)}{\partial t} .
$$

Further, the kernel of the third term's integral on the righthand side of (11) is the longitudinal current change (per unit channel length) due to radial corona current. The spatial integration of (6) and (13) from 0 to $z^{\prime}$ yields the overall longitudinal current change between ground surface and height $z^{\prime}$ at time $t$ due to the upward propagation delay and the radial corona current, respectively. Using (12), we can rewrite (11) for $t \geq z^{\prime} / v$ as follows:

$$
i\left(z^{\prime}, t\right)=i(0, t)-\left[\int_{0}^{z^{\prime}} i_{\text {prop }}^{\prime}(\xi, t) d \xi+\int_{0}^{z^{\prime}} i_{\text {cor }}^{\prime}(\xi, t) d \xi\right]
$$

or

$$
i\left(z^{\prime}, t\right)=i(0, t)-i_{\text {prop }}\left(z^{\prime}, t\right)-i_{\text {cor }}\left(z^{\prime}, t\right)
$$

where $i_{\text {prop }}\left(z^{\prime}, t\right)$ and $i_{\text {cor }}\left(z^{\prime}, t\right)$ are the longitudinal current changes between 0 and $z^{\prime}$ due to upward propagation delay and radial corona current, respectively. Equations (14) and (15) relate the longitudinal return-stroke current at height $z^{\prime}$ and time $t$ to the channel-base current and overall changes in longitudinal current between ground surface and height $z^{\prime}$ at time $t$. Interestingly, the first term on the right-hand side of (15) represents the Bruce-Golde (BG) model, the algebraic sum of the first two terms represents the TL model, and the sum of all three terms represents modified transmission line models [e.g., modified transmission line model with linear current decay with height (MTLL)]. Relations between the BG, TL, and MTLL models are illustrated in Fig. 1.

Note that the new formulation (15) is consistent with the general equation, introduced in [3], which relates the total line charge density $\rho\left(z^{\prime}, t\right)$ to its transferred and deposited components. The total longitudinal current at height $z_{1}=1000 \mathrm{~m}$ for the MTLL model is shown in Fig. 2 as the sum of channelbase current and negated longitudinal current changes due to propagation delay and radial corona current.

\section{Core Charge And Deposited Corona Charge VARIATIONS PREDICTED BY LCS MODELS}

As illustrated below, (15) enables one to make additional inferences about the return-stroke mechanism behind the model. The physical meaning of $i_{\text {prop }}\left(z^{\prime}, t\right)$, which represents the longitudinal current change between channel base and height $z^{\prime}$ at time $t$ due to upward propagation delay, can be better explained using the concept of charges, which are delivered by the current wave from the channel-base source to central core and further to the corona sheath along the channel. First, we take the time integral of both sides of (14) from 0 to $t>z^{\prime} / v$

$$
\left\{\begin{aligned}
\int_{z^{\prime} / v}^{t} i\left(z^{\prime}, \tau\right) d \tau= & \int_{0}^{t} i(0, \tau) d \tau-\int_{0}^{t} \int_{0}^{z^{\prime}} i_{\text {prop }}^{\prime}(\xi, \tau) d \xi d \tau \\
& -\int_{0}^{t} \int_{0}^{z^{\prime}} i_{\text {cor }}^{\prime}(\xi, \tau) d \xi d \tau .
\end{aligned}\right.
$$

We can rewrite (16) as

$$
Q\left(z^{\prime}, t\right)=Q(0, t)-\left[Q_{\text {core }}\left(z^{\prime}, t\right)+Q_{\text {corona }}\left(z^{\prime}, t\right)\right]
$$


BG

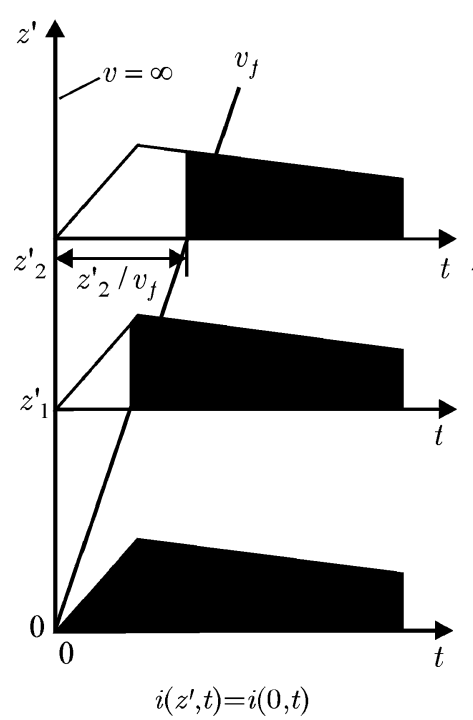

TL

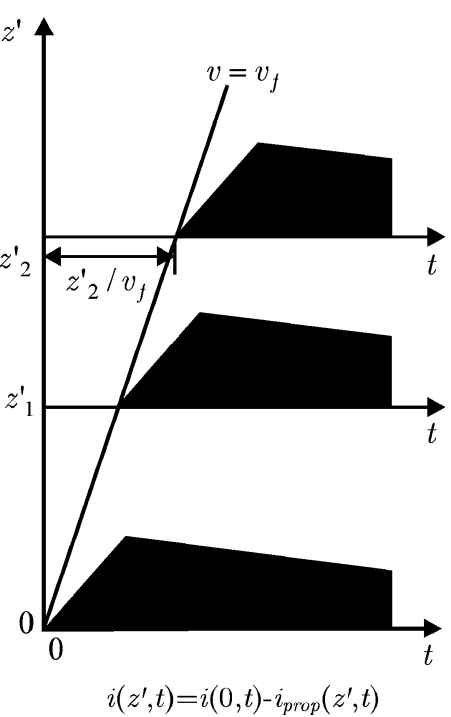

MTLL

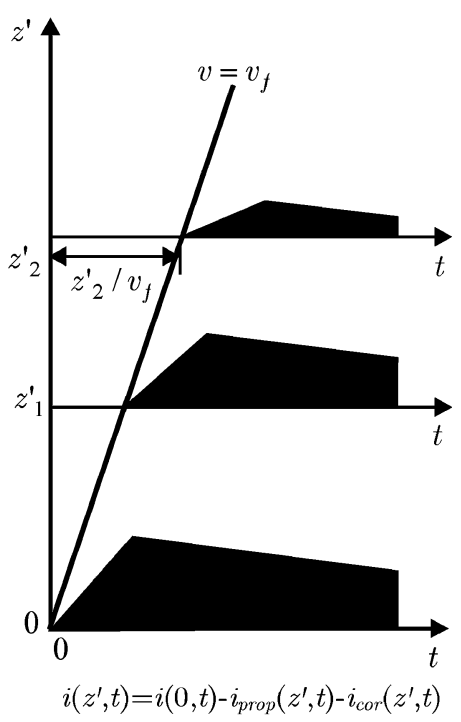

Fig. 1. Current versus time waveforms at ground $z^{\prime}=0$ and two heights $z_{1}^{\prime}$ and $z_{2}^{\prime}$ above ground for the BG, TL, and MTLL return-stroke models. Slanted lines labeled $v_{f}$ represent upward speed of the return-stroke front and lines labeled $v$ represent speed of the return-stroke current wave. The dark portion of the waveform indicates current that actually flows through a given channel section. Note that the current waveforms at $\mathrm{z}^{\prime}=0$ and $v_{f}$ are the same for all three models.

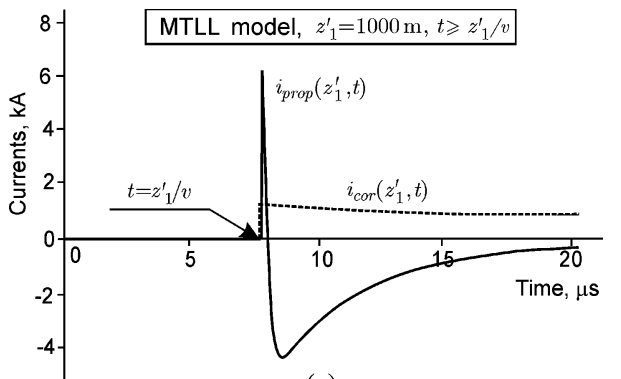

(a)

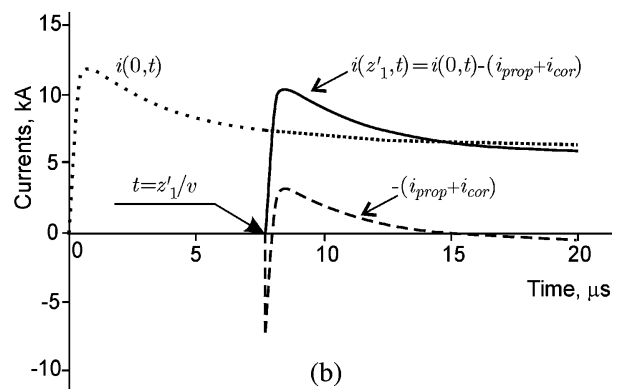

(b)

Fig. 2. (a) Longitudinal current changes due to propagation delay (solid line) and radial corona current (dotted line) between channel base and $z_{1}^{\prime}=1000 \mathrm{~m}$ for the MTLL model $(H=7500 \mathrm{~m}, v=130 \mathrm{~m} / \mu \mathrm{s})$; (b) the longitudinal current $i\left(z_{1}^{\prime}, t\right)$ (solid line) at height $z_{1}^{\prime}=1000 \mathrm{~m}$ and time $t$, channel-base current $i(0, t)$ (dotted line) and negated overall longitudinal current change $-\left(i_{\text {prop }}+i_{\text {cor }}\right)$ (dashed line) between channel base and $z_{1}^{\prime}$. The channel-base current used here is the same as that adopted in [7] and is characterized by a current peak of $12 \mathrm{kA}$ and a maximum current rate of rise of about $40 \mathrm{kA} / \mu \mathrm{s}$.

where $Q\left(z^{\prime}, t\right)$ is the charge transferred upward from height $z^{\prime}$ during the period from $z^{\prime} / v$ to $t, Q(0, t)$ is the charge released by the source at the channel base up to time $t, Q_{\text {core }}\left(z^{\prime}, t\right)$ is the charge contained in the channel core between ground surface and height $z^{\prime}$ at time $t$, and $Q_{\text {corona }}\left(z^{\prime}, t\right)$ is the charge deposited in the corona sheath between ground surface and height $z^{\prime}$ at time $t$. Note that for $v \rightarrow \infty, Q_{\text {core }}=0$ and charge is delivered to height $z^{\prime}$ and to the corona sheath instantaneously. In order to calculate $Q_{\text {core }}$ and $Q_{\text {corona }}$ at time $t$, we have to consider two stages: 1) the time period $0 \leq t<z^{\prime} / v$ when the return stroke front travels between ground and height $z^{\prime}$ and 2) the time period when the return stroke arrives at height $z^{\prime}$ and then moves upward, beyond $z^{\prime}\left(t \geq z^{\prime} / v\right)$. Detailed calculations of the core and corona charges have been described in [5].

For the TL model, it is assumed that the return-stroke waveform travels along the semiinfinite lightning channel without attenuation. Then, the corona current is equal to zero due to the absence of any charge deposited in the corona sheath surrounding the lightning channel core. Shown in Fig. 3 are central core and corona charges contained in the entire lightning channel as a function of time $t$ for the MTLL model.

Also shown in Fig. 3(a) is the variation of the core charge between ground level and height $H$ for the TL model. From this figure, one can deduce that for the TL model, when the current waveform propagates between ground surface and height $H$, the channel core is charged, and then, when the current waveform arrives at height $H$, the channel core is discharged. During the second stage, the charge contained in the core between ground and height $H$ is transferred upward for the TL model, while for the MTLL model the longitudinal current at the channel top $i(H, t)$ is equal to zero and the core charge is spent to neutralize the corona charge deposited by the preceding leader.

Note that the maximum core charge predicted by the TL model is about two times greater than for the MTLL model due to the lack of corona current in the former. For $t \geq H / v$, the core charge between ground surface and height $H$ decreases for both models, and at long enough time is practically equal to zero. According to Fig. 3(b), the corona charge monotonically increases during the return-stroke process up to the value, which is equal to total charge released by the channel-base current source. On the other hand, the core charge increases while the return-stroke 


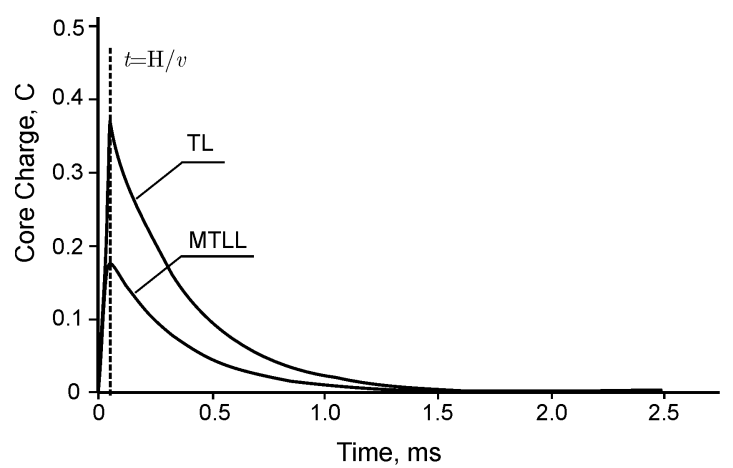

(a)

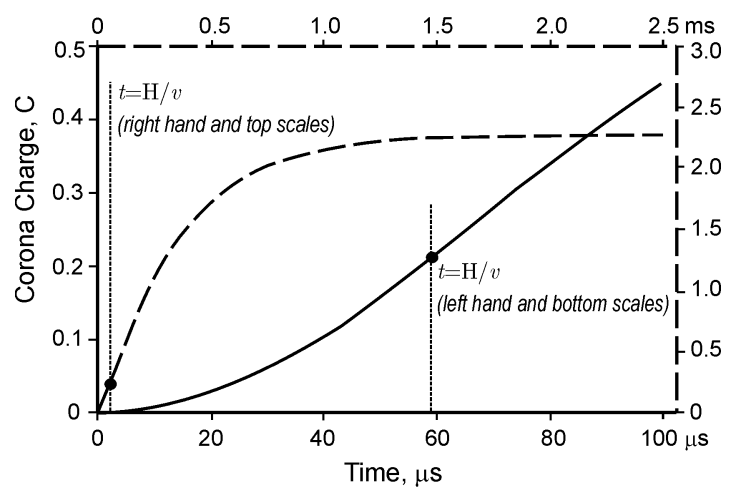

(b)

Fig. 3. (a) Core and (b) corona charges contained in the entire lightning channel as a function of time $t$ for the MTLL model $(H=7500 \mathrm{~m}, v=130 \mathrm{~m} / \mu \mathrm{s})$. Also shown in (a) is the core charge for the TL model, with the corresponding corona charge being equal to zero. The corona charge is shown in (b) on two different time scales, $100 \mu \mathrm{s}$ (solid line) and $2.5 \mathrm{~ms}$ (broken line). The left charge scale corresponds to the bottom time scale and the right charge scale corresponds to the top time scale.

front travels between the ground surface and the channel top (the core is charging) to the maximum value at $t=H / v$, and then decreases to zero as the channel-base current vanishes and the core is discharged. For the original TL model, there is no radial corona and, hence, no longitudinal current change due to corona current. For infinitely large current-wave propagation speed (as assumed in the Bruce-Golde model), there is no propagation delay and, therefore, in this case the longitudinal current change due to upward propagation delay vanishes.

\section{Dynamics of Lightning Channel Corona Sheath}

In Section IV, we analyzed variations in central core charge and deposited corona charge as predicted by LCS lightning return-stroke models. It enables one to consider dynamics of the corona sheath that are implicitly specified by LCS models. In this section, we generalize the corona model introduced in [7]. More information on the role of the corona envelope in various lightning processes is found in [8]. Maslowski and Rakov [7], based on their consideration of the lightning corona sheath dynamics, inferred the existence of two zones around the lightning channel core during the return-stroke stage. The inner zone (Zone 1) has both negative and positive charges with the net charge being positive, and the outer zone (Zone 2) contains only negative charge. The net charge inside the entire corona sheath after the return-stroke stage is equal to zero. A similar electrical structure of the return-stroke corona sheath has been considered in [9], which is concerned with corona processes during the return-stroke stage of long laboratory sparks. In the original corona model of [7], the two-zone charge distribution was assumed to be dissipated by a slower process after the return-stroke stage.

The new corona model includes the motion of negative leader charge from the outer to the inner zone of the corona sheath, toward the core, so that the final neutralization of corona charge occurs during the return-stroke process [10]. In the following, we investigate the corona sheath radius for LCS-type models. In addition, DCS-type models are considered in order to examine the dynamics of the lightning-channel corona sheath and the current generation mechanism during the return-stroke stage that are implied in these models.

\section{A. LCS Models}

The return-stroke current wave traverses the leader channel core and serves to bring it to ground potential. As a result, the leader charge stored in the corona sheath collapses into the channel core and is transferred to ground. The return-stroke process in negative lightning can be visualized as a positive current (and charge density) wave that propagates upward along the leader channel and deposits positive charge in the corona sheath to neutralize the negative charge of the preceding leader.

The deposited charge will create a radial electric field that exceeds the breakdown value and pushes the charge away from the core. The corona sheath expands outward from the channel core until the radial electric field is less than the breakdown value, assumed to be about $2 \mathrm{MV} / \mathrm{m}$ in [11] and $1 \mathrm{MV} / \mathrm{m}$ in [12]. It is generally thought (e.g., [11] and [13]) that the bulk of the leader charge is stored in the corona sheath whose radius is of the order of meters, while the highly conductive channel core (probably less than $0.5 \mathrm{~cm}$ in radius) carries essentially all the longitudinal current.

Consider a closed cylindrical surface (Gaussian cylinder) $d S$ that is coaxial with and surrounding a segment of channel core whose length is $d z^{\prime}$. According to Gauss' law

$$
\varepsilon_{0} \oint_{d S} \mathbf{E} \cdot d \mathbf{S}=d Q
$$

where $\mathbf{E}$ is the electric field on closed surface $d S$ and $d Q$ is the total charge inside this surface. Maslowski and Rakov [7] showed that the contributions of the longitudinal electric field at top and bottom surfaces of the considered cylinder can be neglected and (18) can be rewritten in the equivalent form, as follows:

$$
2 \pi r_{\text {outer }}^{+} \varepsilon_{0} E_{r}^{+} d z^{\prime}=d Q^{+}+d Q^{-}
$$

where $r_{\text {outer }}^{+}$is the outer radius of the inner zone (Zone 1) of corona sheath containing positive charge deposited by the radial conduction current flowing during the return-stroke stage, and $E_{r}^{+}$is the constant radial electric field on the lateral surface of radius $r_{\text {outer }}^{+}$. The total charge $d Q$ enclosed by $d S$ consists of the negative charge $d Q^{-}$deposited by the preceding leader 
and the positive charge $d Q^{+}$associated with the return stroke stage. In order to estimate $r_{\text {outer }}^{+}$, the radial electric field $E_{r}^{+}$ must be chosen. We assume that the corona sheath zone with uniform ionization extends outward from the channel core until the field becomes less than the positive breakdown electric field. Note that the radial electric field cannot be established instantaneously, but we will use a constant value of breakdown field for simplicity. In this paper, we assume that the positive breakdown electric field is equal to $E_{r}^{+}=1 \mathrm{MV} / \mathrm{m}$ [12]. The return-stroke charge $d Q^{+}$can be specified using return-stroke models and represented as the sum of two components [3]

$$
d Q^{+}=\rho_{\text {tran }} d z^{\prime}+\rho_{\mathrm{dep}} d z^{\prime} .
$$

The first term of (20) is the charge transferred upward through the channel segment and the second term represents the deposited charge that is spent to neutralize the leader charge previously deposited in the corona sheath of this segment. Assuming a uniform radial distribution of the negative leader charge just before the return-stroke stage, one can show that $d Q^{-}$, which is a portion of the total negative charge stored in the corona sheath located within the radial extent $r_{\text {out }}^{+}$of positive charge $d Q^{+}$, can be expressed for $t \geq z^{\prime} / v$, as

$$
\begin{aligned}
d Q^{-} & =d Q_{1}^{-}+d Q_{2}^{-} \\
& =k \rho_{L} d z^{\prime}+\left(\rho_{L}-k \rho_{L}\right)\left(1-e^{-\left(t-z^{\prime} / v\right) / \tau_{\mathrm{CN}}}\right) d z^{\prime}
\end{aligned}
$$

where $k=\left(r_{\text {outer }}^{+} / r_{\text {outer }}^{-}\right)^{2}, d Q_{1}^{-}=k \rho_{L} d z^{\prime}$ is the negative leader charge deposited within Zone 1 just before the returnstroke stage, $d Q_{2}^{-}$is the negative charge that penetrates Zone 1 from Zone 2 , and $\tau_{\mathrm{CN}}$ is the decay time constant describing reduction of negative charge deposited within Zone 2, and, hence, the rate of motion of the negative charge from Zone 2 to Zone 1. In fact, it is suggested in [8] that some slow breakdown processes (positive streamers) can develop outward from the uniform breakdown region at fields in excess of as low as 0.2 $\mathrm{MV} / \mathrm{m}$.

According to Appendix B of [7], $d Q_{1}^{-}$can be expressed as

$$
d Q_{1}^{-}=\left(2 \pi \varepsilon_{0} r_{\text {outer }}^{+} E_{r}^{-}\right)^{2} d z^{\prime} / \rho_{L}
$$

where $E_{r}^{-}$is the negative breakdown electric field, which is assumed to be greater (in absolute value) than $E_{r}^{+}$and equal to $1.5 \mathrm{MV} / \mathrm{m}$ [12], and $\rho_{L}$ is the negative charge density per unit channel length prior to the return-stroke stage. Note that $r_{\text {outer }}^{+}$is necessarily smaller than $r_{\text {outer }}^{-}$, the radial extent of the negative leader corona sheath. From (19)-(22) we have

$$
\begin{aligned}
2 \pi r_{\text {outer }}^{+} \varepsilon_{0} E_{r}^{+}= & \rho_{\text {tran }}+\rho_{\text {dep }}+\rho_{L}\left(1-e^{-\left(t-z^{\prime} / v\right) / \tau_{\mathrm{CN}}}\right) \\
& +\frac{\left(2 \pi r_{\text {outer }}^{+} \varepsilon_{0} E_{r}^{-}\right)^{2}}{\rho_{L}} e^{-\left(t-z^{\prime} / v\right) / \tau_{\mathrm{CN}}}
\end{aligned}
$$

The radius of Zone 1, $r_{\text {outer }}^{+}$, is a solution of (23), which reduces to (20) of [7] for $\tau_{\mathrm{CN}} \rightarrow \infty$, in which case negative charge from Zone 2 does not penetrate Zone 1 . The corona sheath model described by (23) enables one to predict both the radial expansion of Zone 1 and its shrinkage up to a nearly zero radius, once the decay time constant $\tau_{\mathrm{CN}}$ is specified (see Fig. 4).

\section{B. DCS Models}

According to [8], the longitudinal return-stroke current can be decomposed into an "extension current" of tens of kiloamperes for tens of microseconds and two "corona currents," one from streamers providing kiloamperes for hundreds of microseconds and the other one from ion drift providing an ampere for seconds. Also, it is suggested in [14] that the return-stroke current can be decomposed into the "breakdown current," which corresponds to the "extension current" introduced in [8], and "corona current." In the following, we will adopt this latter current division. The "breakdown current" is assumed to be generated at the return-stroke tip and in the central core, while the "corona current" originates in the corona sheath surrounding the central core. Under these assumptions, negative line charge density along the lightning channel just before the return-stroke stage $\rho_{\mathrm{L}}\left(z^{\prime}\right)$,can be viewed as the sum of "breakdown," $\rho_{\mathrm{L}}^{\mathrm{BD}}\left(z^{\prime}\right)$, and "corona," $\rho_{\mathrm{L}}^{\mathrm{BD}}\left(z^{\prime}\right)$, components

$$
-\left|\rho_{\mathrm{L}}\left(z^{\prime}\right)\right|=-\left|\rho_{\mathrm{L}}^{\mathrm{BD}}\left(z^{\prime}\right)\right|-\left|\rho_{\mathrm{L}}^{\mathrm{C}}\left(z^{\prime}\right)\right|
$$

where $\rho_{\mathrm{L}}^{\mathrm{BD}}$ is stored in the core and gives rise to the "breakdown current," while $\rho_{\mathrm{L}}^{\mathrm{C}}$ is stored in the corona sheath and is associated with the "corona current." Charge per unit length at height $z^{\prime}$ and time $t \geq z^{\prime} / v$ that is stored in the core is assumed to vary as

$$
-\left|\rho_{\mathrm{L}}^{\mathrm{BD}}\left(z^{\prime}, t\right)\right|=-\left|\rho_{\mathrm{L}}^{\mathrm{BD}}\left(z^{\prime}\right)\right| e^{-\left(t-z^{\prime} / v\right) / \tau_{\mathrm{BD}}}
$$

and the corresponding equation for the corona sheath is

$$
-\left|\rho_{\mathrm{L}}^{\mathrm{C}}\left(z^{\prime}, t\right)\right|=-\left|\rho_{\mathrm{L}}^{\mathrm{C}}\left(z^{\prime}\right)\right| e^{-\left(t-z^{\prime} / v\right) / \tau_{\mathrm{C}}}
$$

where $\tau_{\mathrm{BD}}$ is the breakdown time constant and $\tau_{\mathrm{C}} \gg \tau_{\mathrm{BD}}$ is the corona time constant.

Current $i_{\mathrm{BD}}^{\prime}\left(z^{\prime}, t\right)$ per unit channel length is defined as the time derivative of charge density given by (25), i.e.,

$$
i_{\mathrm{BD}}^{\prime}\left(z^{\prime}, t\right)=\frac{d}{d t}\left(-\left|\rho_{\mathrm{L}}^{\mathrm{BD}}\left(z^{\prime}\right)\right| e^{-\left(t-z^{\prime} / v\right) / \tau_{\mathrm{BD}}}\right)
$$

or

$$
d i_{\mathrm{BD}}\left(z^{\prime}, t\right)=\frac{1}{\tau_{\mathrm{BD}}}\left|\rho_{\mathrm{L}}^{\mathrm{BD}}\left(z^{\prime}\right)\right| e^{-\left(t-z^{\prime} / v\right) / \tau_{\mathrm{BD}}} d z^{\prime} \quad t \geq z^{\prime} / v .
$$

This current is assumed in DCS models to flow downward with the speed of light (see [15]) and is positive according to (28). Similarly

$$
d i_{\mathrm{C}}\left(z^{\prime}, t\right)=\frac{1}{\tau_{\mathrm{C}}}\left|\rho_{\mathrm{L}}^{\mathrm{C}}\left(z^{\prime}\right)\right| e^{-\left(t-z^{\prime} / v\right) / \tau_{\mathrm{C}}} d z^{\prime} \quad t \geq z^{\prime} / v .
$$

We assume that the positive corona current flows with the speed of light into the channel core, and then downward, together with the "breakdown" current, to yield the channel-base current, which is arbitrarily divided into two components associated with the physical processes discussed above. The corresponding physical model and equivalent circuit, which employs a phased shunt current source array, are shown in Fig. 5. According to the assumed division of the channel-base current, described in detail in [14], the breakdown charge is stored primarily in the bottom part of the lightning channel. As a result, the longitudinal 

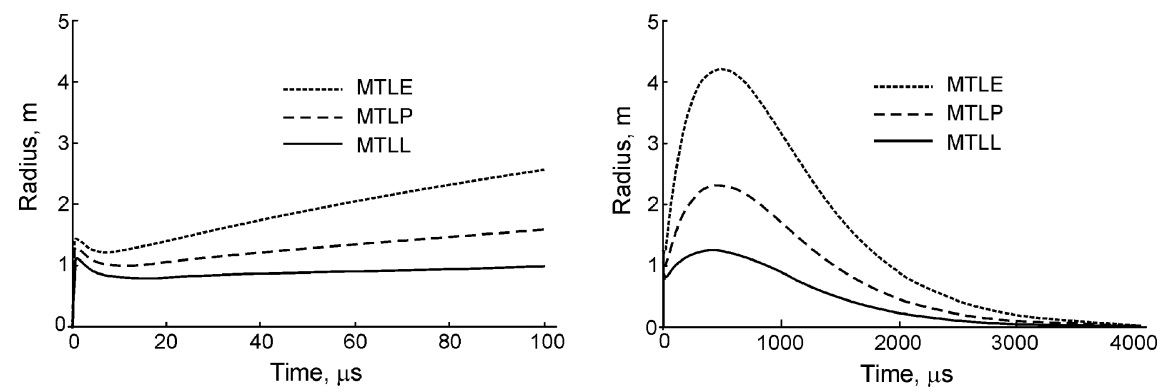

Fig. 4. Comparison of radius $r_{\text {outer }}^{+}$of Zone 1 versus time at a height of $10 \mathrm{~m}$ for three return stroke models, MTLL, modified transmission line model with parabolic current decay with height (MTLP), and MTLE $(H=7500 \mathrm{~m}, \lambda=2000 \mathrm{~m}, v=130 \mathrm{~m} / \mu \mathrm{s}$ ) shown on two different timescales, $100 \mu \mathrm{s}$ (left), and $4 \mathrm{~ms}$ (right), as predicted by (23). It was assumed in (23) that $\tau_{\mathrm{CN}}=650 \mu \mathrm{s}$.

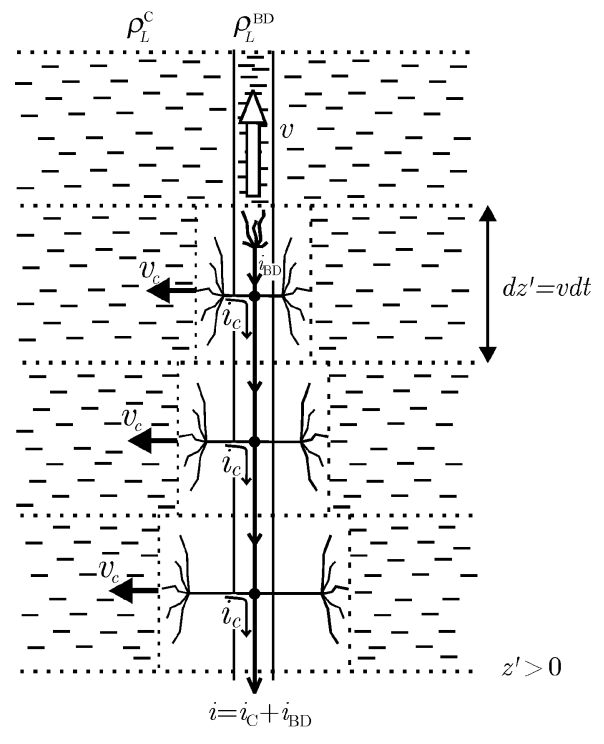

(a)

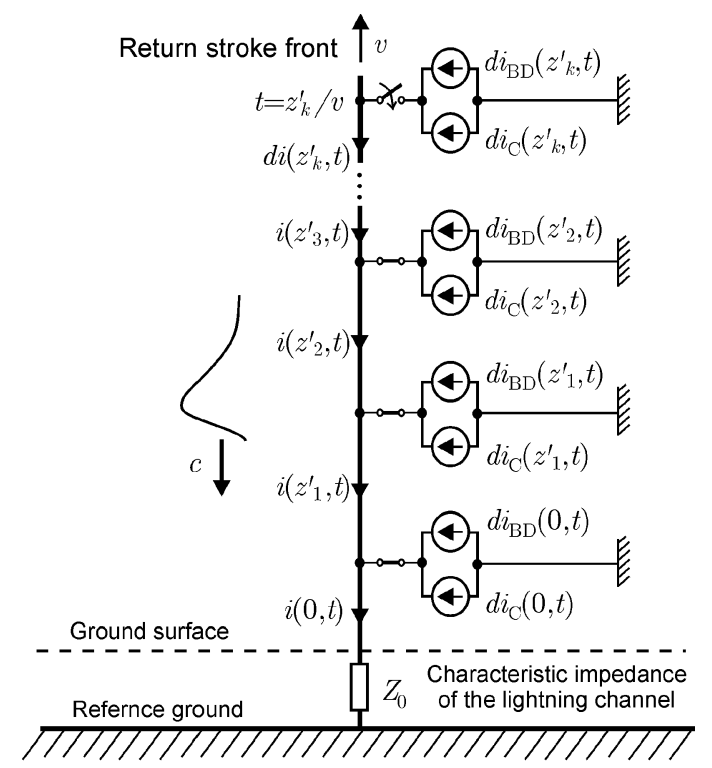

(b)

Fig. 5. (a) Physical model of lightning return-stroke process illustrating the two current components $i_{\mathrm{BD}}$ and $i_{\mathrm{C}}$ in DCS-type models and (b) the equivalent circuit, which employs a phased shunt current source array. At higher altitudes, $i_{\mathrm{BD}} \approx 0$ and $i \approx i_{\mathrm{C}}$.

current in the upper part of the channel is composed primarily of the corona current.

The return-stroke front moves upward with speed $v$ and the "breakdown" current propagates in the opposite direction with the speed of light. Similarly, the return-stroke corona sheath develops radially with speed $v_{\mathrm{c}}$ into the leader corona sheath, and the associated corona current propagates with the speed of light in the opposite direction, into the channel core and then downward to ground (possible propagation toward the upwardmoving front is neglected in DCS models). As a first approximation, one can neglect in (29) the time delay associated with propagation of the corona current inside the sheath. According to Fig. 5(b), two current sources $d i_{\mathrm{BD}}$ and $d i_{\mathrm{C}}$ are progressively activated by the return-stroke front and then these two current components start to flow downward. According to Kirchhoff's law, both current sources $d i_{\mathrm{BD}}$ and $d i_{\mathrm{C}}$ activated at height $z^{\prime}$ give contributions that add to the longitudinal current flowing at height $z^{\prime}$, the latter current being generated by all sources situated above $z^{\prime}$. Note that behind the return-stroke front, the current $d i_{\mathrm{BD}}$ vanishes during several microseconds $\left(\tau_{\mathrm{BD}}=0.6 \mu \mathrm{s}\right)$, while the current $d i_{\mathrm{C}}$ flows much longer.

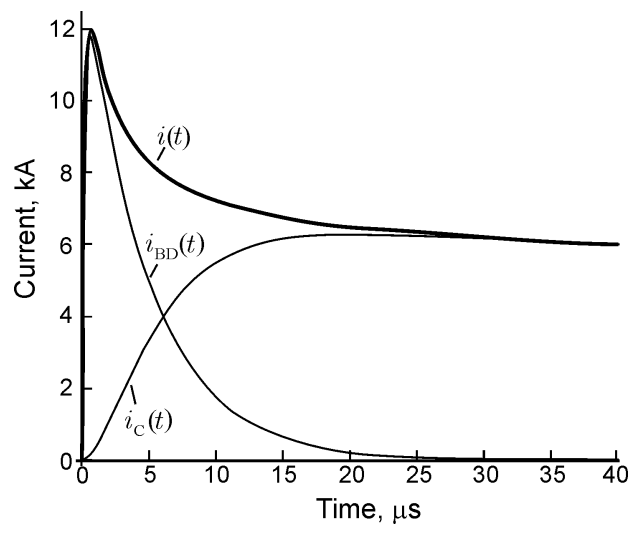

Fig. 6. Illustration of the division of the channel-base current into the "breakdown" and "corona" current components.

Longitudinal current at height $z^{\prime}$ can be calculated in the same way as was done in [14], i.e., by integration of current source contributions $d i_{\mathrm{BD}}$ and $d i_{\mathrm{C}}$ described by (28) and (29), which are distributed along the lightning channel above $z^{\prime}$. As a result, 


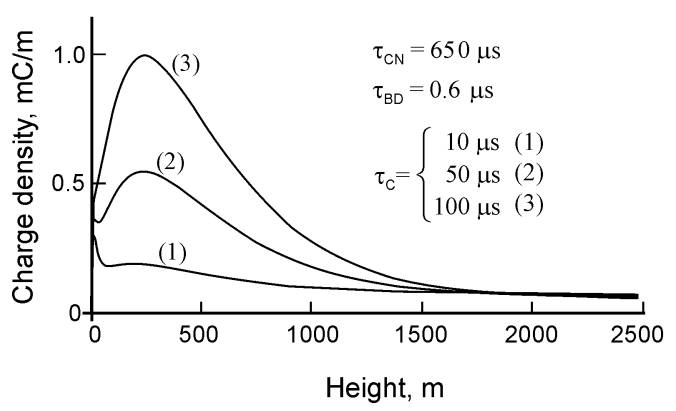

(a)

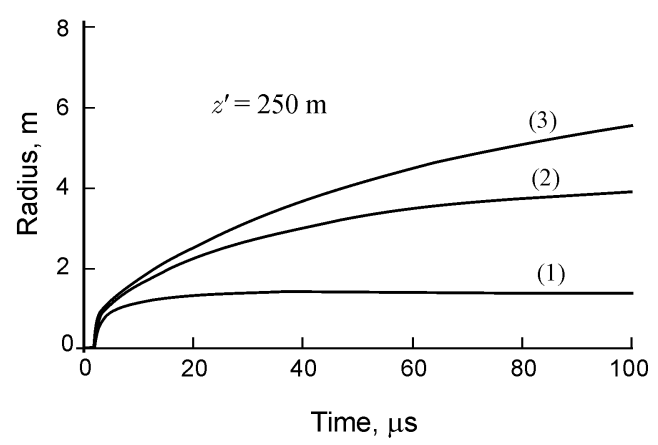

(b)

Fig. 7. Total charge density deposited along the lightning channel during the return-stroke stage (left) and radius of the corona sheath at $z^{\prime}=250 \mathrm{~m}$ versus time in the case of the assumed division of channel-base current (right) for $\tau_{\mathrm{BD}}=0.6 \mu \mathrm{s}, \tau_{\mathrm{CN}}=650 \mu \mathrm{s}$, and three different $\tau_{\mathrm{C}}$. Note that after $58 \mu \mathrm{s}$ the return-stroke front will rise to a height of about $7500 \mathrm{~m}$ (the expected height of negative charge source in the cloud in Florida), which means that model predictions for later time should be viewed with caution.

this current can be described as follows:

$$
\begin{aligned}
i\left(z^{\prime}, t\right)= & i_{\mathrm{BD}}\left(0, t+\frac{z^{\prime}}{c}\right)-i_{\mathrm{BD}}\left(0, \frac{z^{\prime}}{v^{*}}\right) e^{-\left(t-z^{\prime} / v\right) / \tau_{\mathrm{BD}}} \\
& +i_{\mathrm{C}}\left(0, t+\frac{z^{\prime}}{c}\right)-i_{\mathrm{C}}\left(0, \frac{z^{\prime}}{v^{*}}\right) e^{-\left(t-z^{\prime} / v\right) / \tau_{\mathrm{C}}}
\end{aligned}
$$

and at ground level

$$
i(0, t)=i_{\mathrm{BD}}(0, t)+i_{\mathrm{C}}(0, t) .
$$

Following [14], one can also calculate the absolute value of the leader charge density deposited along the channel before the return-stroke stage as a function of the "breakdown" and "corona" currents

$$
\begin{aligned}
\left|\rho_{\mathrm{L}}\left(z^{\prime}\right)\right|= & \frac{1}{v^{*}}\left\{i_{\mathrm{BD}}\left(0, z^{\prime} / v^{*}\right)+\tau_{\mathrm{BD}} \frac{d i_{\mathrm{BD}}\left(0, z^{\prime} / v^{*}\right)}{d t}\right\} \\
& +\frac{1}{v^{*}}\left\{i_{\mathrm{C}}\left(0, z^{\prime} / v^{*}\right)+\tau_{\mathrm{C}} \frac{d i_{\mathrm{C}}\left(0, z^{\prime} / v^{*}\right)}{d t}\right\} .
\end{aligned}
$$

Charge density described by (32) is identical to the deposited charge density at the end of the return-stroke stage, which was obtained in [3]. In fact, for the Diendorfer-Uman (DU) model with two time constants this charge density can be expressed as

$$
\begin{aligned}
& \rho_{\mathrm{L}}\left(z^{\prime}, t\right)=-\frac{i_{\mathrm{BD}}\left(0, t+z^{\prime} / c\right)}{c} \\
& -\left[\frac{i_{\mathrm{BD}}\left(0, z^{\prime} / v^{*}\right)}{v}+\frac{\tau_{\mathrm{BD}}}{v^{*}} \frac{d i_{\mathrm{BD}}\left(0, z^{\prime} / v^{*}\right)}{d t}\right] \\
& \times e^{-\left(t-z^{\prime} / v\right) \tau_{\mathrm{B} \mathrm{D}}} \\
& +\frac{1}{v^{*}}\left[i_{\mathrm{BD}}\left(0, z^{\prime} / v^{*}\right)+\tau_{\mathrm{BD}} \frac{d i_{\mathrm{BD}}\left(0, z^{\prime} / v^{*}\right)}{d t}\right] \\
& -\frac{i_{\mathrm{C}}\left(0, t+\left(z^{\prime} / c\right)\right)}{c} \\
& -\left[\frac{i_{\mathrm{C}}\left(0, z^{\prime} / v^{*}\right)}{v}+\frac{\tau_{\mathrm{C}}}{v^{*}} \frac{d i_{\mathrm{C}}\left(0, z^{\prime} / v^{*}\right)}{d t}\right] \\
& \times e^{-\left(t-z^{\prime} / v\right) \tau_{\mathrm{C}}} \\
& +\frac{1}{v^{*}}\left[i_{\mathrm{C}}\left(0, z^{\prime} / v^{*}\right)+\tau_{\mathrm{C}} \frac{d i_{\mathrm{C}}\left(0, z^{\prime} / v^{*}\right)}{d t}\right] .
\end{aligned}
$$

Note that (33) reduces to (32) for long enough time. It means that (33) represents the positive charge density during the returnstroke stage. Thottappillil et al. [3] proposed division of the total charge density described by (33) into transferred and deposited charge density components. Cooray [6] modified the original division proposed in [3]. According to Cooray's division (adopted in this paper), for $t \geq z^{\prime} / v$, the transferred charge density for two time constants can be written as

$$
\begin{aligned}
\rho_{\mathrm{tran}}\left(z^{\prime}, t\right) & =-\frac{i_{\mathrm{BD}}\left(0, t+z^{\prime} / c\right)}{c}+\frac{i_{\mathrm{BD}}\left(0, z^{\prime} / v^{*}\right)}{c} e^{-\left(t-z^{\prime} / v\right) \tau_{\mathrm{B}}} \\
& -\frac{i_{\mathrm{C}}\left(0, t+z^{\prime} / c\right)}{c}+\frac{i_{\mathrm{C}}\left(0, z^{\prime} / v^{*}\right)}{c} e^{-\left(t-z^{\prime} / v\right) \tau_{\mathrm{C}}}
\end{aligned}
$$

and deposited charge density as

$$
\begin{aligned}
\rho_{\mathrm{dep}}\left(z^{\prime}, t\right)= & \frac{\tau_{\mathrm{BD}}}{v^{*}}\left\{\frac{d i_{\mathrm{BD}}\left(0, z^{\prime} / v^{*}\right)}{d t}+\frac{i_{\mathrm{BD}}\left(0, z^{\prime} / v^{*}\right)}{\tau_{\mathrm{BD}}}\right\} \\
& \times\left[1-e^{\left.-\left(t-z^{\prime} / v\right) / \tau_{\mathrm{BD}}\right]}\right. \\
& +\frac{\tau_{\mathrm{C}}}{v^{*}}\left\{\frac{d i_{\mathrm{C}}\left(0, z^{\prime} / v^{*}\right)}{d t}+\frac{i_{\mathrm{C}}\left(0, z^{\prime} / v^{*}\right)}{\tau_{\mathrm{C}}}\right\} \\
& \times\left[1-e^{-\left(t-z^{\prime} / v\right) / \tau_{\mathrm{C}}} .\right.
\end{aligned}
$$

Deposited charge density at height $z^{\prime}$ described by (35) is zero for $t=z^{\prime} / v$ and for $t>z^{\prime} / v$ increases up to a value, which is consistent with (32).

Now, we can use (23) to investigate dynamics of the lightning channel corona sheath predicted by DCS-type models, in which $\rho_{\text {tran }}, \rho_{\text {dep }}$, and $\rho_{\mathrm{L}}=-\left|\rho_{\mathrm{L}}\left(z^{\prime}\right)\right|$ are described by (34), (35), and (32), respectively. We assume the same channel-base current as in [7]. This current can be arbitrarily divided into two current components (see Fig. 6), this division being similar to that in [14].

Using these current components, one can calculate the line charge density deposited along the lightning channel at the end of the return stroke stage and radius of the corona sheath versus time for different discharge time constants.

According to Fig. 7(a), the distribution of the total charge density along the channel strongly depends on the time constant $\tau_{\mathrm{C}}$. For greater $\tau_{\mathrm{C}}$, more charge has to be deposited near the 
bottom of the channel in order to satisfy the specified channelbase current.

In the case of the assumed division of channel-base current and relatively small $\tau_{\mathrm{C}}$, the maximal line charge density is predicted close to the ground surface [see curve (1) in Fig. 7(a)] and for greater $\tau_{\mathrm{C}}$, the maximal line charge density is at a height of about $250 \mathrm{~m}$ [see curves (2) and (3) in Fig 7(a)]. Radii of the corona sheath at $z^{\prime}=250 \mathrm{~m}$ for three different values of $\tau_{\mathrm{C}}$ are shown in Fig. 7(b).

\section{SUMMARY}

Any lightning return-stroke model with specified longitudinal current distribution can be expressed, using the appropriate continuity equation, in terms of two current components, propagation current and corona current. For LCS-type models, the actual corona current is unipolar and directed radially out of the channel core, while for DCS-type models, it is also unipolar but directed into the channel core. The conversion between LCS and DCS return-stroke models alters the actual corona current (if any) of the model. A new formulation for LCS lightning return-stroke models is proposed using corona current and propagation current concepts. This formulation expresses the longitudinal current at height $z^{\prime}$ as the channelbase current minus the overall longitudinal current change from ground surface to $z^{\prime}$. Dynamics of lightning-channel corona sheath surrounding the thin channel core are examined based on the LCS and DCS models that specify different corona currents.

\section{ACKNOWLEDGMENT}

This research was supported in part by NSF. The authors would like to thank F. Rachidi for his comments on the paper.

\section{REFERENCES}

[1] V. A. Rakov and M. A. Uman, Lightning: Physics and Effects. Cambridge, U.K.: Cambridge Univ. Press, 2003.

[2] V. A. Rakov and A. A. Dulzon, "A modified transmission line model for lightning return stroke field calculation," in Proc. 9th Int. Zurich Symp. Electromagn. Compat., Zurich, Switzerland, 1991, pp. 229-235.

[3] R. Thottappillil, V. A. Rakov, and M. A. Uman, "Distribution of charge along the lightning channel: Relation to remote electric and magnetic fields and to return-stroke models," J. Geophys. Res., vol. 102, pp. 6987-7006, 1997.

[4] G. Maslowski and V. A. Rakov, "Equivalency of lightning return-stroke models employing lumped and distributed current sources," IEEE Trans. Electromagn. Compat., vol. 49, no. 1, pp. 123-132, Feb. 2007.

[5] G. Maslowski and V. A. Rakov, "A new formulation for lightning returnstroke models of engineering type," in Proc. 18th Int. Zurich Symp. Electromagn. Compat., Munich, Germany, 2007, pp. 175-178.

[6] V. Cooray, "On the concepts used in return stroke models applied in engineering practice," IEEE Trans. Electromagn. Compat., vol. 45, no. 1, pp. 101-108, Feb. 2003.

[7] G. Maslowski and V. A. Rakov, "A study of the lightning channel corona sheath," J. Geophys. Res., vol. 111, D14110, 2006. Doi: 10.1029/2005 JD006858.

[8] S. J. Heckman and E. R. Williams, "Corona envelopes and lightning currents,” J. Geophys. Res., vol. 94, pp. 13287-13294, 1989.

[9] B. N. Gorin, "Mathematical modeling of the lightning return stroke," Elektrichestvo, vol. 4, pp. 10-16, 1985.

[10] G. Maslowski, V. A. Rakov, J. Cvetic, and M. Miki, "An improved model for prediction of the dynamics of lightning channel corona sheath," in
Proc. 20th Int. Zurich Symp. Electromagn. Compat., Zurich, Switzerland, 2009, pp. 121-124.

[11] C. E. Baum and L. Baker, "Analytic return-stroke transmission-line model," in Lightning Electromagnetics, R. L. Gardner, Ed. New York: Hemisphere, 1990, pp. 17-40.

[12] V. Kodali, V. A. Rakov, M. A. Uman, K. J. Rambo, G. H. Schnetzer, J. Schoene, and J. Jerauld, "Triggered lightning properties inferred from measured currents and very close electric fields," Atmos. Res., vol. 75, pp. 335-376, 2005.

[13] V. A. Rakov and M. A. Uman, "Review and evaluation of lightning return stroke models including some aspects of their application," IEEE Trans. Electromagn. Compat., vol. 40, no. 4, pp. 403-426, Nov. 1998.

[14] G. Diendorfer and M. A. Uman, "An improved return stroke model with specified channel-base current," J. Geophys. Res., vol. 95, pp. 13621$13644,1990$.

[15] F. Heidler, "Traveling current source model for LEMP calculation," in Proc. 6th Int. Zurich Symp. Electromagn. Compat., Zurich, Switzerland, 1985, pp. 157-162.

[16] M. Miki, V. A. Rakov, K. J. Rambo, G. H. Schnetzer, and M. A. Uman, "Electric fields near triggered lightning channels measured with Pockels sensors," J. Geophys. Res., vol. 107, pp. D11-D16, 2002.

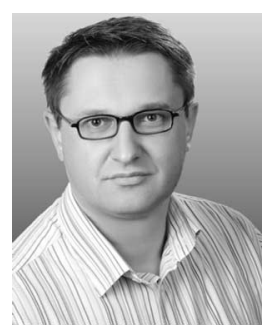

Grzegorz Maslowski (M'08) received the M.S. degree in electrical engineering from the Rzeszow University of Technology (Rzeszow Polytechnic), Rzeszow, Poland, 1991, and the Ph.D. degree from the University of Mining and Metallurgy, Krakow, Poland, in 1999.

Since 1991, he has been with the Department of Electrical and Computer Engineering, Rzeszow University of Technology. He was a Visiting Scholar at the University of Bologna in 2003, and at the University of Florida, Gainesville, in 2005 and 2006. He is the author or coauthor of 50 publications on lightning and its effects. His current interests include the modeling of processes in the lightning flash, lightning protection, and signal processing.

Dr. Maslowski is a member of the American Geophysical Union, the Polish Committee on Lightning Protection, and the Working Group 2 of the COST Action P18: The Physics of Lightning Flash and Its Effects. He is also a member of the Board of Reviewers of the Biennial International Zurich Symposium on Electromagnetic Compatibility.

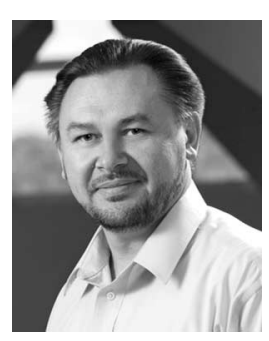

Vladimir A. Rakov (SM'96-F'03) received the M.S. and Ph.D. degrees in electrical engineering from the Tomsk Polytechnical University (Tomsk Polytechnic), Tomsk, Russia, in 1977 and 1983, respectively.

From 1977 to 1979, he was an Assistant Professor of electrical engineering at Tomsk Polytechnic. From 1978, he was engaged in lightning research at the High Voltage Research Institute (a division of Tomsk Polytechnic), where from 1984 to 1994, he was the Director of the Lightning Research Laboratory. He is currently a Professor at the Department of Electrical and Computer Engineering, University of Florida, Gainesville, and Co-Director of the International Center for Lightning Research and Testing (ICLRT). He is the lead author of one book and author or coauthor of over 500 other publications on various aspects of lightning, with more than 160 papers being published in reviewed journals.

Prof. Rakov is the Chairman of the Technical Committee on Lightning of the Biennial International Zurich Symposium on Electromagnetic Compatibility and former Chairman of the American Geophysical Union (AGU) Committee on Atmospheric and Space Electricity. He is a Fellow of the American Meteorological Society and The Institution of Engineering and Technology and a member of the AGU. 